\title{
Cost-effective Migration towards Virtualized C-RAN with Scalable Fronthaul Design
}

\author{
Meysam Masoudi*, Shari Sofia Lisi*, Cicek Cavdar* \\ ${ }^{*}$ School of Electrical Engineering and Computer Science, KTH Royal Institute of Technology, Stockholm, Sweden \\ Email: \{masoudi,sslisi,cavdar\}@kth.se
}

\begin{abstract}
Migration from distributed to centralized radio access networks (C-RANs) can be expensive in terms of capital expenditures due to the initial investment while it has lower operational expenditures due to pooling baseband processing into the cloud and reduced power consumption. Partial centralization can be also an option by employing network function splitting and keeping lower physical layer functions co-located with the radio units. This increases the power consumption but relaxes the high capacity requirement in the fronthaul. It is not intuitive which migration strategy is more cost effective. In this paper, we formulate a pool placement optimization problem as an integer linear programming (ILP), which minimizes the total cost of ownership (TCO), and evaluate the migration cost to C-RAN with both full centralization of network functions, and partial centralization by using function splitting. We define a network upgrade optimization problem, by adding new cells to the network, as a revisited version of the original optimization problem to evaluate the upgradability of the architectures. We solve the problem with both ILP for optimality, and genetic algorithm for scalability. Simulation results show that partial centralization results in optimal TCO with lower crossover time compared to C-RAN with full centralization.
\end{abstract}

Index Terms-Total cost of ownership, functional splitting, CRAN, integer linear programming, genetic algorithm.

\section{INTRODUCTION}

\section{A. Motivation}

Radio access networks (RAN) are facing a rapid increase in traffic demand, due to increasing number of devices connected to the network, and higher quality of service requested by the users. Network operators must be able to provide the required capacity to fulfill such demand. C-RAN has been proposed as a promising architecture to increase network capacity, while improving energy efficiency, and providing scalability [1]. In a traditional RAN, the components constituting the base station are located at the cell site, and are divided into a radio unit (RU) for the transmission and reception of radio signals, and a digital unit (DU) for the baseband signal processing. The main idea of C-RAN is to centralize the digital units in a shared location responsible for coordinated signal processing and management, which is called DU pool, while keeping the radio units at the cell sites. Such centralization allows maintenance costs and energy consumption to be shared among several digital units. To transport the baseband signals between the radio units and the digital units, a low latency aggregation network, known as fronthaul, is utilized [2]. There are different proposals for the fronthaul architecture such as millimeter wave fronthaul and time and wavelength division multiplexing passive optical networks (TWDM-PON). TWDMPON is a promising architecture to support the low latency high capacity requirement of fronthaul transport network [3]. Although full centralization of network functions in C-RAN reduces both operational expenditures $(\mathrm{OpEx})$, in terms of energy consumption and maintenance, and capital expenditure (CapEx), in terms of new site deployments, cost of the fronthaul is a challenge. Therefore, to moderate the rigorous capacity requirements for fronthaul, the network function processing can be distributed between the RUs and the cloud. However, the profitability of migrating to the new architecture is still questionable. In this study we attempt to assess the migration cost of current network to the C-RAN with TWDM-PON architecture.

\section{B. Literature Study}

Despite the attractive advantages of C-RAN, its deployment brings considerable challenges for the operators. The progress on C-RAN as well as its major challenges are discussed in [4]. When all the network functionalities in the base band unit $(\mathrm{BBU})$ are fully centralized, C-RAN may require approximately $160 \mathrm{~Gb} / \mathrm{s}$ of throughput with $10 \mu \mathrm{s}-250 \mu \mathrm{s}$ of latency [5]. If lower physical layer (PHY) functions are kept co-located with the RUs, these requirements become looser. For instance, the fronthaul transport link requirement for lower level PHY functional, e.g., split 4 in Figure 1, is about $933 \mathrm{Mbps}$ which is only about $40 \%$ of a fully centralized scenario. Figure 1 shows that the baseband processing functions contain a chain of functions that are categorized into cell-processing (CP) and user-processing (UP) functions. CP functions are dedicated to signal processing and physical layer functions, e.g., serial to parallel, common public radio interface (CPRI) encoding, and resource demapping. UP functions are dedicated to per user signal processing, e.g., equalization and turbo coding. Using functional splitting in C-RAN, it is possible to split this processing chain at certain points [6]. The requirements for the fronthaul link between the cloud and radio unit depend on the functional split point. Maximum benefit in terms of energy saving and virtualization is attained when all the functions are centralized at the cloud which is equivalent to traditional C-RAN. However, this might not be possible due to the limited fronthaul capacity, hence using different split points supported by the cost-effective fronthaul is more preferable [7]. In Figure 1, the possible split points are depicted and in Table I the capacity requirements in the fronthaul links are 


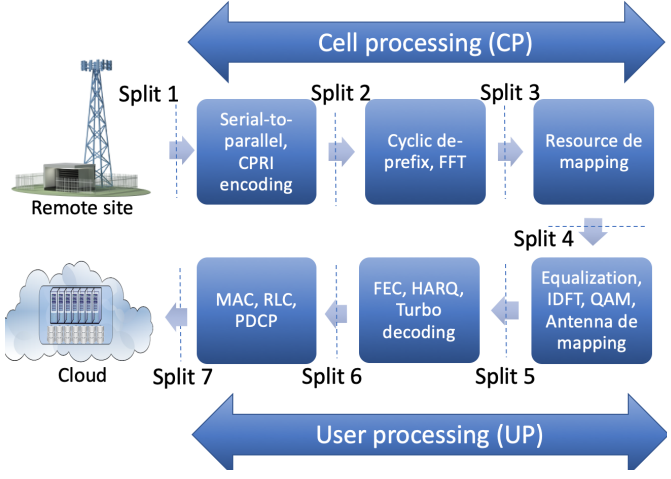

Figure 1: Functional split model

summarized [7], [8]. These requirements make the passive optical networks the most suitable transport medium in the fronthaul [9]. Therefore, from the design perspective new challenges should be considered. These challenges include, (i) optimal placement of pools to centralize the processing, (ii) optimal design of fronthaul to guarantee the requirements, and (iii) cost of the network. To tackle such challenges, the authors

Table I: Fronthaul capacity requirement for different split points

\begin{tabular}{|c|c|c|c|c|c|c|c|}
\hline & \multicolumn{7}{|c|}{ Capacity requirements (Mbps) } \\
\hline Split point: & 7 & 6 & 5 & 4 & 3 & 2 & 1 \\
\hline Downlink & 151 & 151 & 173 & 933 & 1075 & 1966 & 2457.6 \\
Uplink & 48 & 49 & 452 & 903 & 922 & 1966 & 2457.6 \\
\hline
\end{tabular}

in [10] proposed an energy efficient aggregation network and a pool placement optimization problem, to minimize the total aggregation infrastructure power. The authors in [11] proposed an optimization problem to determine the configuration of each DU pool, which minimizes the deployment cost. The fronthaul constraint, which must guarantee a certain latency, the infrastructure deployment, and the pool placement, are among the main challenges of C-RAN. The authors in [12] presented an overview of the fronthaul requirements, and proposed architectures and transmission technologies for next generation optical access networks. The authors in [13] addressed the fronthaul constraint problem by proposing several solutions such as signal compression and quantization, coordinated signal processing, and radio resource allocation optimization. The study in [14] investigated the impact of wired and wireless backhaul on the delay performance of cellular networks. In their study, the infrastructure cost of backhaul deployment was also considered.

In order to assess the monetary aspect of the network, a thorough cost model of the network is required. The authors in [15] modeled the power consumption of backhaul with different scenarios and investigated the impact of the backhaul network to TCO in cellular networks. They demonstrated that if a power efficient backhaul solution such as fiber optic architecture is adopted, the share of backhauling power consumption will be small compared to the radio access part. The study in [16] attempted to model the total cost of backhauling with two different technologies, i.e., microwave and fiber. Based on this study, it was shown that fiber is the most promising backhaul technology with high capacity and low delay.
To provide a techno-economic framework of C-RAN architecture, all the network costs must be considered. Therefore, the studies [17]-[23] take into account TCO as their evaluation metric. In [17], the authors presented a methodology to evaluate TCO for different technologies. Their study mainly focused on migration cost of optical access technologies considering infrastructure and technology upgrade. The authors in [18] proposed a TCO model that considers scalability, CapEx and OpEx, as well as the uncertainty in different stages such as demand and penetration index. They have shown that according to resource designation, there is a great sensitivity in the maximum expected benefit. The authors in [19] analyzed the TCO of network migration towards passive optical network, concerning both infrastructure and technology upgrades. In their study, they considered different migration starting times, customer penetration, node consolidation, and network provider business roles in the fiber access networks. The study in [20] presented a comprehensive techno-economic framework able to assess both the TCO and the business viability of different deployments. The authors stated that TCO alone is not sufficient to understand the profitability of different architectures and more parameters such as net present value (NPV) and cash flow should be considered. The study in [21] modeled the costs of fiber and microwave architectures and calculated the TCO for different geographical regions. In their study, the optimal fronthaul design and functional splitting is missing.

In [22], the authors investigated the migration of LTE networks to C-RAN with minimal investment. They proposed an ILP-based algorithm to migrate to the C-RAN architecture with minimal investment by employing the available infrastructure. In their study, they quantified the economic advantages of using the available infrastructure while migrating to C-RAN. They compared two migration scenarios with and without using the available infrastructure. Their results indicated that significant savings that can be obtained by using the currently installed infrastructure. The study in [23] formulated TCO minimization problem as a constraint programming problem. In this study, the authors found the optimal functional split point for radio unit and digital unit pair. However, the problem of finding optimal location for pools was not resolved. The study in [24] provided a cost analysis of the deployment of the wireless and optical x-haul segment for a fixed wireless access network. They considered RAN functional splits with diverse requirements capacity and coverage requirements. In [25], we investigated the cost efficient migration towards C-RAN and proposed an ILP model to solve the pool placement problem optimizing for energy and cost. In this study, we compared the TCO of C-RAN with microwave and optical distributed RAN (D-RAN) over time. We also compared two different approaches for the network design: 1) energy efficient and 2) cost efficient approach. We indicated that energy efficient solutions are not necessarily cost efficient due to necessity of initial investments for centralization. We have also investigated the impact of cell size on the TCO of C-RAN networks. In a long term, we found that the smaller the cell size is, the more cost efficient C-RAN becomes with respect to D-RAN. In our preliminary study, functional splitting is not considered and the ILP solution is not scalable for large problem sizes. 


\section{Contribution}

In this paper, the migration cost from today's D-RAN to C-RAN is evaluated, with the flexibility of functional splitting. To this end, the total cost of C-RAN is determined, and a DU pool placement optimization problem is defined to minimize such cost. The total cost of C-RAN is modeled according to the concept of TCO, which is defined as the sum of build-out costs, i.e. CapEx, and operation and maintenance costs plus electric bills, i.e. OpEx. The TCO of C-RAN is compared over time to that of traditional D-RAN, which is initially assumed to only include OpEx. This analysis is intended to understand the migration cost of C-RAN, and the amount of time needed to compensate for such cost, under different cost assumptions. However, as capacity demands are forecast to increase, a further deployment of base stations will be needed. A second analysis is carried out to understand the upgradability of C-RAN. A network upgrade optimization problem is defined which minimizes the TCO of upgrading the network. This analysis is intended to understand and compare the upgrade capabilities of C-RAN and D-RAN, in terms of TCO. In our model, we merge deployment cost and energy consumption into a single quantity, while related work has only considered the energy consumption [10] or the deployment cost [11]. With our framework, one can optimally design C-RAN and make an informed decision regarding the migration from the existing architecture. The main contributions of this paper are as follows:

- The formulation of a TCO with the flexibility of functional splitting, to identify the migration cost of C-RAN.

- The formulation of DU pool placement problem and a network upgrade optimization problem to minimize the TCO, as an ILP problem.

- We propose a scalable meta-heuristic based on genetic algorithm (GA) to solve the problem. Our solution has the ability to handle medium and large problem sizes and gives near optimal solutions.

- Investigating the impact of functional splitting on the TCO.

- Investigating the sensitivity of TCO with regards to the price fluctuations.

The remainder of this paper is organized as follows. Section II presents the network architecture and power models. Section III discusses the business assessment of the network. The DU pool placement optimization problem is presented in Section IV. Section IV-E presents the network upgrade optimization problem. In Section V, we propose a heuristic algorithm to solve the problem. Simulation results are reported in Section VI. Conclusions are summarized in Section VII.

\section{Network ARchitecture}

In this section, we describe the network architecture and present the TCO model. First, we identify the components needed to implement C-RAN, which affect the TCO and, consequently, the optimization problem. Then, we present D-RAN architecture, to represent today's networks as a baseline. Power consumption models for the two aforementioned architectures

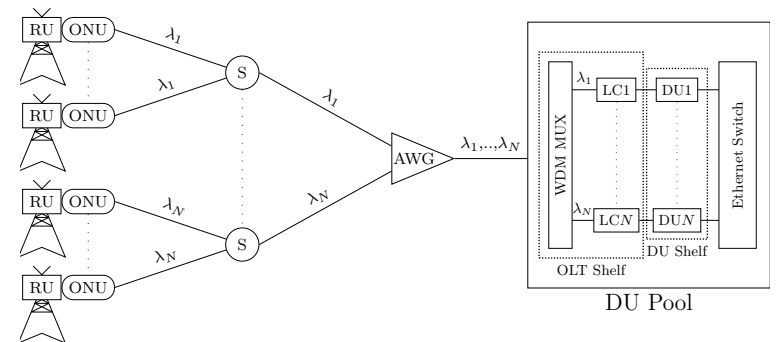

Figure 2: TWDM-PON fronthaul architecture.

are defined. These models are necessary for the formulation of the TCO model at the end of this section.

\section{A. C-RAN Architecture}

In this paper, C-RAN fronthaul is assumed to be a TWDMPON. This means that both wavelength and time division multiplexing are performed at different network layers, to exploit the bandwidth efficiency of WDM with the cost efficiency of TDM. We assume two aggregation levels in the fronthaul. The first aggregation level is composed of passive splitters, denoted as $\mathrm{S}$, while the second aggregation level is composed of arrayed wavelength grating (AWG) filters. The radio units access the first aggregation level in time division, and the second aggregation level in wavelength division, denoted as $\lambda_{i}$. The TWDM-PON fronthaul architecture is illustrated in Figure 2.

The two main components are the optical network unit (ONU), located at the cell site, and the optical line terminal (OLT), located at the pool site. These components perform electrical to optical signal conversion and vice versa. The OLT considered in this architecture provides single line rates of 10 Gbps, and it is composed of a wavelength division multiplexer, and a line card (LC) for each DU. The CPRI standard sets a constraint on the round trip time between radio units and digital units, in order to achieve seamless baseband signal transmission. This constraint translates to a maximum ONUOLT distance between 20 and $40 \mathrm{~km}$ [1]. Splitting ratios of both AWG filters and splitters must be defined. We denote $N$ and $M$ as the AWG and splitter splitting ratio, respectively. In the definition of splitting ratios, we must consider the line rate constraint imposed by the CPRI standard. For a system where radio units are provided with LTE coverage of 3 sectors with $20 \mathrm{MHz}$ bandwidth and 2x2 MIMO configuration, the minimum line rate must be set to 2.4576 Gbps [26]. Thus, a maximum number of four CPRI flows can be transmitted in a $10 \mathrm{Gbps}$ link, which leads to $M_{\max }=4$. We assume that a fiber can achieve a maximum rate of $80 \mathrm{Gbps}$, i.e. the number of wavelengths per fiber in the TWDM-PON is at maximum $N_{\max }=8$ [27]. Since the fronthaul capacity may not be enough to support the data transmission in $5 \mathrm{G}$ networks (assuming fully centralized scenario), distributing the processing functions between RUs and the cloud can alleviate the burden on the fronthaul. Figure 1 represents the possible split points and Table I shows the capacity requirement by each split point. For instance, split 1 corresponds to the case where all functions are centralized at the cloud, while split 7 to the 
case where all the processing is performed at the RUs, which is equivalent to the D-RAN architecture. In [23], all split points are investigated and it is shown that split 4 and split 6 are more viable. In this study, we assume a fixed split point, i.e., split 4 , because it mitigates the fronthaul capacity requirements while allowing to benefit from PHY layer techniques such as joint transmission/reception coordinated multipoint (CoMP) [7]. According to Table I, if split 4 is chosen, the minimum line rate must be set to $933 \mathrm{Mbps}$. Therefore, a maximum number of ten flows can be transmitted in 10 Gbps link, which leads to $M_{\max }=10$. We assume both greenfield and brownfield deployment of fiber. In the greenfield case, existing D-RAN with microwave backhaul is assumed. Therefore, the optical infrastructure must be deployed through digging and roll out operations. In the brownfield case, existing D-RAN with fiber backhaul is assumed, hence the optical infrastructure is already partially deployed. We reduce the fiber digging and roll out costs by a scaling factor $\alpha$ to capture a variety of fiber deployment scenarios.

\section{B. D-RAN Architecture}

D-RAN architecture is assumed to have either microwave or optical backhaul. We will benchmark our work with respect to both types of infrastructure. The microwave backhaul is composed of microwave links connecting the cell sites to the central office (CO) through aggregation nodes, namely microwave hubs [15]. We assume that each hub is composed of four sectors, and each sector supports up to six cell sites, with a maximum capacity of 1.2 Gbps per sector. Furthermore, we assume that the $\mathrm{CO}$ supports a maximum of 96 microwave links. The optical backhaul is assumed to be TWDM-PON, as in the case of C-RAN architecture.

\section{Power Consumption Model}

According to the deployed architecture, i.e. C-RAN, microwave D-RAN, and optical D-RAN, we derive different power models, as follows.

1) C-RAN: The power consumption of C-RAN is defined as the sum of three components, namely the base station power consumption $P_{B S}$, the ONU power consumption $P_{O N U}$, and the DU pool power consumption $P_{P o o l}$. It is expressed as

$$
P_{C R A N}=N_{B S}\left(P_{B S}+P_{O N U}\right)+N_{\text {Pool }} P_{\text {Pool }},
$$

where $N_{B S}$ denotes the number of base stations, and $N_{P o o l}$ the number of DU pools. The base station power consumption $P_{B S}$ is defined as

$$
P_{B S}=N_{T X}\left(P_{o}+\Delta p P_{\text {Out }}\right)+P_{\text {Proc }}^{B S},
$$

where $N_{T X}$ denotes the number of transmitters per base station, $P_{o}$ the power consumption of the base station calculated at minimum transmitted power, $\Delta p$ the slope of the load dependent power consumption, $P_{O u t}$ the maximum power consumption of the base station above the $P_{o}$ [28], and $P_{P \text { roc }}^{B S}$ the power consumption of DUs located at the BSs. The latter is defined as, $P_{P r o c}^{B S}=P_{D U}^{B S} N_{D U}^{B S}$, where $P_{D U}^{B S}$ is the power consumption of a DU, and $N_{D U}^{B S}$ is the number of required active DUs at the BS. The term $N_{D U}^{B S}$ is the function of split point and required giga operation per second (GOPS).

The DU pool power consumption is defined as

$$
P_{\text {Pool }}=N_{D U}\left(P_{\text {Proc }}+P_{O L T}\right)+P_{C o o l},
$$

where $N_{D U}$ denotes the number of digital units per pool, $P_{P r o c}$ the processing power consumption of each digital unit, $P_{O L T}$ the power consumption of each OLT module, and $P_{C o o l}$ the power consumption of the air cooling unit.

2) Optical D-RAN: The power consumption of optical DRAN is defined as

$$
P_{O D}=N_{B S}\left(P_{B S}+P_{P r o c}+P_{C o o l}+P_{O N U}+P_{O L T}\right)(4)
$$

Each base station has dedicated processing and air cooling units, and the OLT modules in the CO are not shared among several radio units.

3) Microwave D-RAN: The power consumption of microwave D-RAN is defined as the sum of three components, namely the power consumption at the cell site, the aggregation node power consumption $P_{A g}$, and the backhaul link power consumption $P_{B h l}$, as in [15]. It is expressed as

$$
P_{m d}=N_{B S}\left(P_{B S}+P_{\text {Proc }}+P_{C o o l}\right)+P_{A g}+P_{B h l} .
$$

The aggregation node power consumption is defined as

$$
\begin{aligned}
P_{A g} & =\beta P_{m, A g}+\frac{N_{B h l} R_{B S}}{R_{A g}}(1-\beta) P_{m, A g} \\
& +N_{U L} P_{U L}+N_{D L} P_{D L},
\end{aligned}
$$

where $P_{m, A g}$ denotes the maximum power supplied to the aggregation node, $\beta$ the percentage of $P_{m, A g}$ consumed in idle mode, $N_{B h l}$ the number of backhaul links, $R_{B S}$ the maximum backhaul load offered by each base station, assumed to be $0.05 \mathrm{Gbps}$, and $R_{A g}$ the capacity of the aggregation node, assumed to be $3.6 \mathrm{Gbps}$ [15]. While $N_{D L}$ denotes the number of downlink interfaces in the aggregation node, $P_{D L}$ the power consumed in downlink, $N_{U L}$ the number of uplink interfaces in the aggregation node, and $P_{U L}$ the power consumed in uplink. The backhaul link power consumption is defined as

$$
P_{B h l}=P_{o, B h l}+\frac{R_{B S}}{C_{B h l}} P_{m, B h l},
$$

where $P_{o, B h l}$ denotes the backhaul link power consumption at minimum transmitted power, and $P_{m, B h l}$ the maximum transmitted power above $P_{o, B h l} . C_{B h l}$ denotes the maximum capacity of the backhaul link and is assumed to be 1.2 Gbps [15]. Table II reports the power values of the aforementioned parameters.

Table II: Power consumption parameters [25].

\begin{tabular}{llll}
\hline Parameter & Value $[\mathrm{W}]$ & Parameter & Value $[\mathrm{W}]$ \\
\hline$P_{o}$ & 53 & $P_{O L T}$ & 20 \\
$\Delta p$ & 3.1 & $\beta$ & 0.6 \\
$P_{O u t}$ & 6.3 & $P_{m, A g}$ & 57 \\
$P_{P r o c}$ & 100 & $P_{U L}$ & 2 \\
$P_{D S}^{B S}$ & 50 & $P_{D L}$ & 1 \\
$P_{C o o l}$ & 500 & $P_{o, B h l}$ & 97 \\
$P_{O N U}$ & 3.4 & $P_{m, B h l}$ & 37 \\
\hline
\end{tabular}




\section{Business ASSESSMENTS OF THE ARChiteCtURE}

The lifecycle of communication networks consists of 4 phases namely, planning, installation, operation, and migration. Since in the migration phase we may require to deploy new architecture, it is important to evaluate the profitability of the deployment to reduce the risk of investment [20]. In this section, we present a techno-economic framework to assess the cost of C-RAN and D-RAN architectures.

\section{A. Total Costs of Ownership (TCO)}

The TCO covers both the CapEx and OpEx. CapEx refers to the costs of building up a network while OpEx refers to the network's operation costs. Depending on the architecture, TCO components might have different terms. For instance, the cost for CapEx covers the infrastructure and equipment (installation and purchase) expenses. The cost for the OpEx covers energy, renting, maintenance, spectrum, leasing, and fault management expenses. The summary of different cost components are summarized in Figure 3. According to the deployed architecture, i.e. C-RAN, microwave D-RAN, and optical D-RAN, we derive different TCO models, as follows.

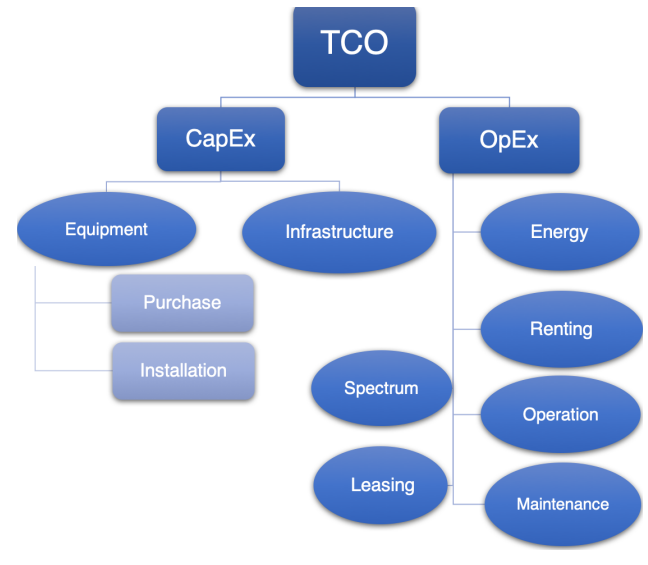

Figure 3: Network cost summary

1) C-RAN: The TCO of C-RAN is expressed as the sum of CapEx and OpEx. The CapEx is defined as the sum of pool build-out, equipment, fiber digging and roll out costs. The build-out costs are not included in the brownfield pool case. The OpEx is defined as the sum of operation and maintenance costs, pool site rent, fiber lease, and electric bills. Cost values for CapEx and OpEx (electric bills excluded) are reported in Table III. The annual electric bills are derived from the power model by assuming $E l_{\text {price }}=1 \frac{€}{\text { Watt }}$. TCO for CRAN can be calculated as:

$$
\begin{aligned}
T C O & =N_{q}\left(C_{\text {build }}+E l_{\text {price }} P_{\text {Pool }}+C_{\text {rent }}\right) \\
& +N_{B S}\left(E l_{\text {price }}\left(P_{B S}+P_{\text {ONU }}\right)\right)+N_{B S} C_{\text {onu }} \\
& +L_{f}\left(C_{\text {dig }}+C_{\text {rol }}\right)+N_{\text {split }} C_{\text {split }}+N_{\text {oltm }} C_{\text {oltm }} \\
& +N_{\text {olt }} C_{\text {olt }}+N_{\text {awg }} C_{\text {awg }}+N_{\text {fiber }} C_{\text {lease }}+C_{\text {oem }}
\end{aligned}
$$

where $N_{q}$ is number of pools, $N_{\text {fiber }}$ is the number of fiber links, and $L_{f}$ is the total fiber length. All other parameters and values are defined in Tables II and III. The first line in (8) is the pool expenses, the second line is the cost of base stations and the remaining are the costs due to architecture equipment and maintenance.

2) Optical D-RAN: The TCO of optical D-RAN includes only OpEx, hence operation and maintenance cost, cell site rent, fiber lease, and electric bills. TCO for optical D-RAN can be formulated as:

$T C O=E l_{\text {price }}\left(P_{O D}\right)+N_{\text {fiber }} C_{\text {lease }}+C_{\text {rent }}+C_{\text {oem }}$

where $P_{O D}$ is defined in (4) and all other parameters and values are defined in Tables II and III.

3) Microwave D-RAN: Similar to optical D-RAN, the TCO of microwave D-RAN includes only OpEx, hence operation and maintenance costs, cell site rent, spectrum fee, and electric bills. TCO for microwave D-RAN can be formulated as:

$$
\begin{aligned}
T C O & =E l_{\text {price }} P_{m d}+W N_{\text {Link }} C_{\text {spec }}+N_{B h l} C_{\text {link }} \\
& +N_{s w} C_{s w}+N_{h u b} C_{h u b}+C_{\text {rent }}+C_{\text {oem }}
\end{aligned}
$$

where $W$ is the bandwidth, $N_{\text {link }}$ is number of the links, $C_{\text {spec }}$ is the annual spectrum fee per MHZ, $N_{B h l}$ is number of backhaul links, $C_{\text {link }}$ is the microwave link cost, $N_{s w}$ and $C_{s w}$ are the number and costs of microwave switches, $N_{h u b}$ and $C_{h u b}$ are the number and costs of hub site. $P_{m d}$ is defined in (5) and the values for microwave TCO calculation are defined in Table IV.

\section{B. Cost Models and Pricing}

The cost model consists of different components, whose values are subject to fluctuations over time. For instance, the price of equipment usually decreases in time due to new technologies and also technology maturation. On the other hand, the human resources expenses such as salaries typically increase over the time. Therefore, price variation is important and should be taken into account when calculating the expenses. There are different approaches to capture price fluctuation in time. For instance, a learning curve can be used in the industry to predict the reduction of the cost of a product [29]. This is a valid approach provided that the right learning curve is used which is not an easy task. In our study, the cost variation is calculated as [20], $C_{j}=C_{0}+\eta C_{j-1}$, where $C_{j}$ denotes the cost in year $j$ of the network operational time, $C_{0}$ is the initial cost, and $\eta$ denotes the cost change factor. Typically, $\eta<0$ for the equipment and hardware components, and $\eta>0$ for the salaries and energy costs. In general, $\eta$ might also vary over time. However, for simplicity in our study $\eta$ is assumed to be constant during the network operational time. The cost values coincide with the ones of C-RAN and D-RAN architectures reported in Tables III and IV, where it is assumed that the cell site and pool site rents are identical.

\section{Net Present Value (NPV)}

The most commonly used evaluation tool to assess investment decisions is the NPV method computed as

$$
N P V=\sum_{t=0}^{T} \frac{C F_{t}}{(1+b)^{t}},
$$


Table III: CapEx and OpEx of C-RAN [25].

\begin{tabular}{lll}
\hline Component & Parameter & Cost $[\mathrm{k} €]$ \\
\hline DU pool build-out & $C_{\text {build }}$ & 75 \\
Equipment: & & \\
- ONU & $C_{\text {onu }}$ & 0.25 \\
- Passive splitter & $C_{\text {split }}$ & 0.1 \\
- AWG & $C_{\text {awg }}$ & 0.25 \\
- OLT access module & $C_{\text {oltm }}$ & 6.5 \\
- OLT shelf & $C_{\text {olt }}$ & 35 \\
Fiber digging per km & $C_{\text {dig }}$ & 60 \\
Fiber rollout per km & $C_{\text {rol }}$ & 3.8 \\
Annual operation and maintenance & $C_{\text {oem }}$ & $10 \%$ of equipment \\
Annual DU pool rent & $C_{\text {rent }}$ & 8 \\
Annual fiber lease & $C_{\text {lease }}$ & 3 \\
\hline
\end{tabular}

Table IV: OpEx of microwave D-RAN [25].

\begin{tabular}{lll}
\hline Component & Parameter & Cost [k€] \\
\hline Microwave link & $C_{l i n k}$ & 0.4 \\
Microwave hub site, 24 ports & $C_{h u b}$ & 0.8 \\
10 Gbit Ethernet Switch, 24 ports & $C_{\text {sw }}$ & 0.18 \\
Annual cell site rent & $C_{r e n t}$ & 8 \\
Annual spectrum fee per MHz/link & $C_{\text {spec }}$ & 0.06 \\
\hline
\end{tabular}

where $b$ denotes the discount rate, $T$ is the time period of evaluation, and $C F_{t}$ is the cash flow at the end of year $t$. Cash flow is defined as the difference between TCO and income of the same year. In particular, if the NPV is positive, the deployment is profitable. On the other hand, if the NPV is negative, the deployment generates a monetary loss in the period of assessment. Since in our study the income is assumed to be the same for all architectures, we modify the NPV expression as,

$$
N P V=\sum_{t=0}^{T} \frac{-T C O_{t}}{(1+b)^{t}},
$$

In this case, we compare the total costs of network over the period of $\mathrm{T}$ years for different architectures. Given the income for each year, it is possible to evaluate the profitability of each network. It is worth mentioning that maximizing NPV corresponds to minimizing TCO which is our objective.

\section{Sensitivity Analysis}

A complementary method to enrich the NPV method is sensitivity analysis. Sensitivity analysis provides a framework that measures the level of confidence in the NPV outcome. It takes a systematic approach to determine the impact of an input variable's variation on the output of the analysis. It can also determine that the investment decision might not be the same under all circumstances. A shortcoming of sensitivity analysis is that it depends on the ability to predict all possible states and variations. Nevertheless, it gives a broader insight for decision making than with only NPV [30].

\section{DU Pool Placement Optimization Problem}

In this section, the DU pool placement optimization problem is presented. Given a set of radio units, the goal is to find the optimal number and location of pools, digital units, and splitters, such that all the radio units are connected to a pool and the TCO is minimized. For simplicity, the AWG are assumed to be located in the same locations as the pools, hence they are not included in the optimization problem. The pool is considered as a shared location where the digital units are stacked on each other, according to the model presented in [31]. The digital units coincide with the processing units residing at the cell site in a traditional distributed topology. This is an ILP problem and is formulated as follows:

Given: set of radio units and digital units; candidate locations of pools and splitters; capacity of pools, digital units, AWG and splitters; maximum distance allowed between pools, splitters and radio units; cost and energy consumption values.

Find: optimal locations of pools, digital units, and splitters; connections between pools, splitters, and radio units.

Objective: minimize the TCO.

Below, the input parameters, decision variables, objective function and constraints are listed.

\section{A. Input Parameters}

$Q$ : set of pools

$D$ : set of digital units

$S$ : set of splitters

$U$ : set of radio units

$F: Q \times S$ distance matrix whose element $f_{q s}$ denotes the distance from pool $q$ to splitter $s$

$G: S \times U$ distance matrix whose element $g_{s u}$ denotes the distance from splitter $s$ to radio unit $u$

$D_{\text {cpri }}$ : maximum distance between pool and splitter

$D_{\text {split }}$ : maximum distance between splitter and radio unit

$C_{\text {pool }}$ : capacity of the pool in terms of digital units

$C_{\text {split }}$ : capacity of the splitter in terms of radio units

$B$ : a large positive number, to enforce the integrality constraint of the decision variables

\section{B. Binary Decision Variables}

$x_{q}=1$ if pool $q$ is active

$y_{d}=1$ if digital unit $d$ is active

$n_{s}=1$ if splitter $s$ is active

$w_{q d}=1$ if digital unit $d$ is connected to pool $q$

$l_{q d s}=1$ if splitter $s$ is connected to digital unit $d$ and pool $q$

$m_{q d s u}=1$ if radio unit $u$ is connected to splitter $s$, digital unit $d$ and pool $q$

\section{Objective Function}

The objective is the minimization of the TCO, expressed as

$$
\begin{aligned}
& \min \left\{\sum_{q \in Q} x_{q}\left(C_{\text {build }}+C_{\text {rent }}\right)+L_{f}\left(C_{\text {dig }}+C_{\text {rol }}\right)\right. \\
& +N_{B S} C_{\text {onu }}+N_{\text {olt }} C_{\text {olt }}+N_{\text {split }} C_{\text {split }}+N_{\text {awg }} C_{\text {awg }} \\
& +N_{\text {oltm }} C_{\text {oltm }}+C_{\text {oem }}+C_{\text {lease }} N_{\text {fiber }} \\
& \left.+E l_{\text {price }}\left(N_{B S}\left(P_{B S}+P_{\text {ONU }}\right)+\sum_{q \in Q} x_{q} P_{\text {Pool }}\right)\right\} .
\end{aligned}
$$

The first line of (13) denotes the total build-out costs and fiber costs, respectively. The build-out costs are zero for brownfield pool deployment. The term $L_{f}$ denotes the total fiber length, 
i.e., the sum of the total length between pools and splitters, and between splitters and radio units, expressed as

$$
L_{f}=\sum_{q \in Q} \sum_{d \in D} \sum_{s \in S} f_{q s} l_{q d s}+\sum_{q \in Q} \sum_{d \in D} \sum_{s \in S} \sum_{u \in U} g_{s u} m_{q d s u} \text {. }
$$

The terms in above equation are defined in Section IV-A and IV-B. The second and third lines of (13) denote the equipment costs. The fourth line denotes operation and maintenance costs, pool site rent, and fiber lease, respectively. The fifth line denotes the electric bills from the power consumption. The terms $N_{\text {split }}$, $N_{\text {awg }}, N_{\text {olt }}$, and $N_{\text {oltm }}$ denote the total number of splitters, AWG, OLT shelves and OLT modules, respectively.

\section{Constraints}

Distance constraints:

$$
\begin{aligned}
& f_{q s} l_{q d s} \leq D_{c p r i} \\
& \forall q \in Q, \forall d \in D, \forall s \in S \\
& g_{\text {su }} m_{q d s u} \leq D_{\text {split }} \quad \forall q \in Q, \forall d \in D, \forall s \in S, \forall u \in U
\end{aligned}
$$

Capacity constraints:

$$
\begin{array}{ll}
\sum_{d \in D} w_{q d} \leq C_{\text {pool }} & \forall q \in Q \\
\sum_{q \in Q} \sum_{d \in D} \sum_{u \in U} m_{q d s u} \leq C_{\text {split }} & \forall s \in S
\end{array}
$$

Binary constraints:

$$
\begin{array}{ll}
\sum_{q \in Q} w_{q d} \leq 1 & \forall d \in D \\
\sum_{q \in Q} \sum_{s \in S} l_{q d s} \leq 1 & \forall d \in D \\
\sum_{q \in Q} \sum_{d \in D} l_{q d s} \leq 1 & \forall s \in S \\
\sum_{q \in Q} \sum_{d \in D} \sum_{s \in S} m_{q d s u}=1 & \forall u \in U
\end{array}
$$

Activity constraints:

$$
\begin{array}{ll}
B x_{q} \geq \sum_{d \in D} w_{q d} & \forall q \in Q \\
x_{q} \leq \sum_{d \in D} w_{q d} & \forall q \in Q \\
B y_{d} \geq \sum_{q \in Q} \sum_{s \in S} l_{q d s} & \forall d \in D \\
y_{d} \leq \sum_{q \in Q} \sum_{s \in S} l_{q d s} & \forall d \in D \\
B n_{s} \geq \sum_{q \in Q} \sum_{d \in D} \sum_{u \in U} m_{q d s u} & \forall s \in S \\
n_{s} \leq \sum_{q \in Q} \sum_{d \in D} \sum_{u \in U} m_{q d s u} & \forall s \in S
\end{array}
$$

Connection constraints:

$$
\begin{array}{lr}
B w_{q d} \geq \sum_{s \in S} l_{q d s} & \forall q \in Q, \forall d \in D \\
w_{q d} \leq \sum_{s \in S} l_{q d s} & \forall q \in Q, \forall d \in D \\
B l_{q d s} \geq \sum_{u \in U} m_{q d s u} & \forall q \in Q, \forall d \in D, \forall s \in S \\
l_{q d s} \leq \sum_{u \in U} m_{q d s u} & \forall q \in Q, \forall d \in D, \forall s \in S
\end{array}
$$

Constraint (15) limits the maximum distance between pools and splitters, while Constraint (16) limits the maximum distance between splitters and radio units. These distances are set to satisfy the CPRI constraint. Constraint (17) and Constraint (18) limit the maximum number of digital units that a pool can host, and the maximum number of radio units that a splitter can serve, respectively. Constraints (19) and (20) enforce each digital unit to be associated to exactly one pool and one splitter, respectively. Constraint (21) enforces each active splitter to be associated to exactly one digital unit, while Constraint (22) enforces each radio unit to be connected to exactly one splitter. Constraints (23) and (24) ensure the selection of a pool, if there is at least one digital unit connected to it. Similarly, Constraints (25) and (26) ensure the activation of a digital unit, if there is at least one splitter connected to it. Constraints (27) and (28) ensure the placement of a splitter, if there is at least one radio unit connected to it. Constraints (29) and (30) enforce the connection between a pool and a digital unit, if there is a splitter connected to both. Finally, Constraints (31) and (32) enforce the connection between a pool, a digital unit, and a splitter, if there is a radio unit connected to all of them. $B$ is inserted to activate the corresponding constraints when it is necessary. B can not be a small number since it makes the constraints too tight and can not be very large since the constraints would be too loose, which would cause implementation problems.

\section{E. C-RAN Upgrade Optimization Problem}

In this section, the upgrade optimization problem is defined as a revisited version of the DU pool optimization problem. The main idea is to exploit the already deployed equipment when possible, and therefore reduce the upgrade cost. Hence, while the structure of the problem does not change, some input parameters must be modified, to take into account existing pools, splitters and fiber connections. Specifically, pools and splitters capacities must be updated for the existing pools and splitters, and the cost for fiber connections between already existing pools and splitters must be set to zero. Given $C_{\text {pool }}$ and $C_{\text {split }}$ initial capacities of pools and splitters, respectively, and given $w_{q d}$ and $m_{q d s u}$ decision variables from the DU pool placement optimization problem, new pools and splitters capacities $\bar{C}_{p o o l, q}$ and $\bar{C}_{s p l i t, s}$ for the upgrade optimization problem are defined as follows:

$$
\begin{array}{r}
\bar{C}_{\text {pool }, q}=C_{\text {pool }}-\sum_{d \in D} w_{q d} \quad \forall q \in Q, \\
\bar{C}_{s p l i t, s}=C_{s p l i t}-\sum_{q \in Q} \sum_{d \in D} \sum_{u \in U} m_{q d s u} \forall s \in S,
\end{array}
$$


where the capacities are decremented depending on the number of DUs already assigned to pools, and radio units already assigned to splitters. The fiber costs for already existing connections between pools and splitters must be set to zero. For simplicity, we set the distance from the related pools and splitters $\bar{f}_{q s}$ to zero instead, as follows:

$$
\bar{f}_{q s}= \begin{cases}0 & \text { if } l_{q d s}=1 \\ f_{q s} & \text { otherwise }\end{cases}
$$

Hence, Constraint 15, Constraint 17, and Constraint 18 are rewritten as follows:

$$
\begin{aligned}
& \bar{f}_{q s} l_{q d s} \leq D_{\text {cpri }} \quad \forall q \in Q, \forall d \in D, \forall s \in S \\
& \sum_{d \in D} w_{q d} \leq \bar{C}_{\text {pool }} \quad \forall q \in Q \\
& \sum_{q \in Q} \sum_{d \in D} \sum_{u \in U} m_{q d s u} \leq \bar{C}_{\text {split }} \quad \forall s \in S
\end{aligned}
$$

The resulting TCO includes the extra costs needed to deploy the new cells, hence extra CapEx and OpEx.

\section{Genetic Algorithm to Solve the Problem}

Although an ILP based approach gives an optimal solution, it suffers from scalability issues. For instance, in our study the ILP based approach does not scale well with number of pools larger than 25 . Since real-world planning problems are commonly larger, there is a need to develop a scalable solution which can handle larger problem sizes. In this section, genetic algorithm is utilized to solve the fronthaul design problem. However it is worth mentioning that, while the advantage of heuristic based approaches is their scalablilty, they cannot guarantee the optimality of solutions [32].

\section{A. Individual Design}

In GA, at each run solutions are encoded as a set of individuals (or chromosomes). Better individuals have higher chance to survive and mimic themselves more often in the reproduction phases, namely mutation and crossover. For the network design, we encode the individuals as depicted in Figure $4 \mathrm{a}$. In the individual encoder, rows represent pools' locations and columns represent the radio units. The intersection of rows and columns (pools and RUs) consists of a vector showing the DU activation. If any of the DUs are active, then the corresponding pool location becomes active and the active DU is responsible for serving the RU. Additionally, knowing the number of active RUs in each pool gives the number of active digital units, since each digital unit has limited capacity of serving RUs. Furthermore, the required number of splitters to implement such a network can be obtained by figuring out the number of active DUs, RUs, and pools. It is worth mentioning that some constraints such as distance constraint are considered when the individuals are selected, therefore, the calculated number of active splitters are based on feasible solutions and hence we do not consider all of them in the individual formation phase.

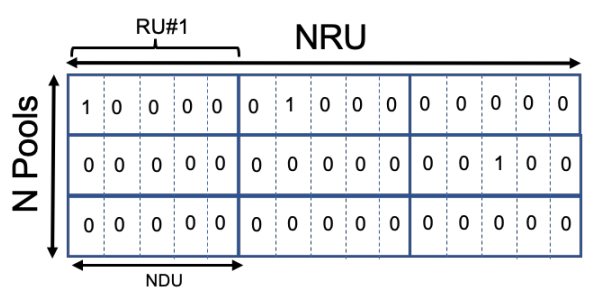

(a) The individual encoder.

Mutation

\begin{tabular}{|lllllllllllllllll|l|l|}
\hline 0 & 0 & 0 & 0 & 0 & 0 & 0 & 0 & 0 & 0 & 0 & 0 & 0 & 0 & 0 \\
\hline \hline 1 & 0 & 0 & 0 & 0 & 0 & 0 & 0 & 1 & 0 & 0 & 0 & 0 & 0 & 0 \\
\hline 0 & 0 & 0 & 0 & 0 & 0 & 0 & 0 & 0 & 0 & 0 & 0 & 1 & 0 & 0 \\
\hline
\end{tabular}

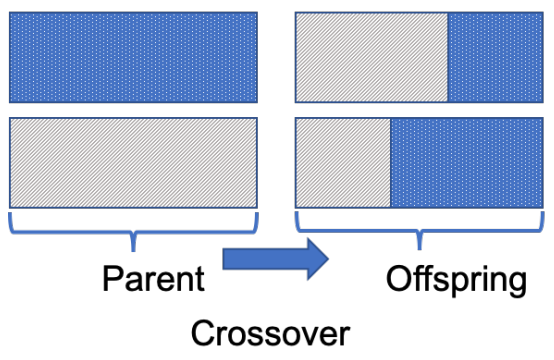

(b) The mutation and crossover phase example

Figure 4: An encoder example for 3 RUs, 3 pools, and 5 DUs per pool. RU 1 and 2 are connected to DU 1 and 2 in Pool 1 respectively, pool 2 serves RU 3 and Pool 3 is inactive.

\section{B. GA procedure}

In this section the algorithm steps are explained. The pseudocode of genetic algorithm is provided in Algorithm 1. This algorithm illustrates the procedure of generating solutions and different phases of GA. In the first iteration, the initial population is generated randomly based on Figure $4 \mathrm{a}$.

In crossover phase, with probability $p_{c}$, individuals are selected. Then partial genes of parent's individual are exchanged to reproduce new offsprings. In this phase, from the network design perspective, new association of RUs-Pools are generated. Since the new solution or design are based on previous designs, it may be a better solution; however, some of these offsprings are infeasible solutions and will be discarded later.

Mutation is used to avoid falling and remaining into the local optimum. In the mutation phase, multiple points of the individual are modified randomly which means new subset of RUs-Pools associations are randomly added to the individuals. In order not to be stuck with the local optimum here, few infeasible individuals are allowed. Thanks to the mutation phase, continuously new and different designs are created with the hope of finding better and different network design. Elitism in GA means keeping the best generated individuals intact for the next generation. To consider the elitism, we first sort the solutions based on i) feasibility, ii) objective (for feasible solutions), and iii) the amount of condition violation (for infeasible solutions). Then the algorithm picks the $N_{p}$ best solutions. These solutions will replace the initial solutions and the algorithm continues until the convergence criteria is met. 


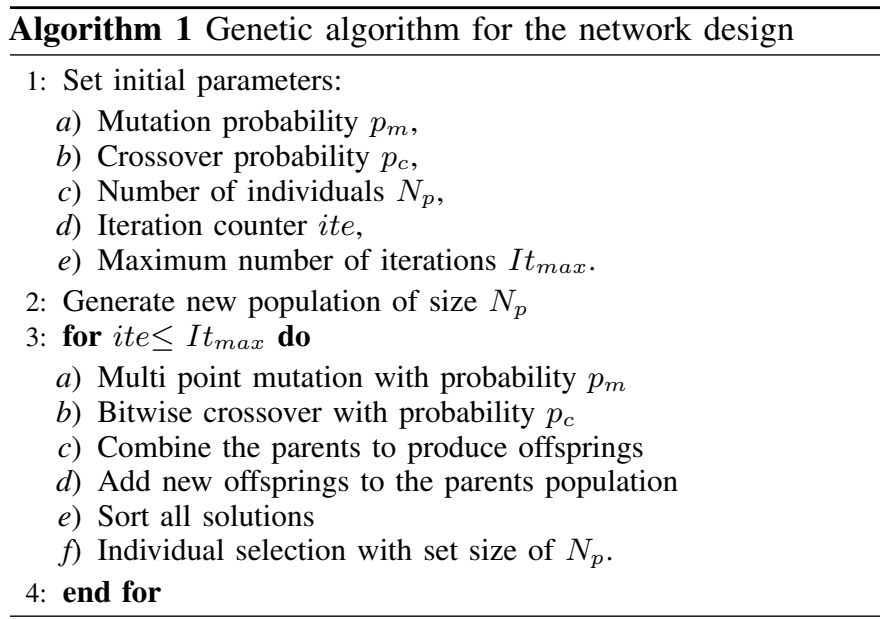

\section{Simulation Results}

In this section, we solve the DU pool optimization problem using IBM ILOG CPLEX Optimization Studio and genetic algorithm. The former can solve the ILP problem optimally but is not scalable while the latter is scalable and can be used for larger network size. The drawback of the GA is that it does not guarantee the optimality of the solutions. Hence, in this section, we first compare the GA solutions with the optimal solutions to investigate the optimality gap, and then we compare the resulting TCO of C-RAN scenarios with D-RAN, to assess the migration cost of C-RAN.

We assume that a pool can host a maximum of 48 digital units for the greenfield pool case, and a maximum of 4 digital units for the brownfield pool case. We assume that one DU at the BS can serve all the processing functions related to the serving BS. For instance, in case of fully centralized scenario, e.g., C-RAN, $N_{D U}^{B S}=0$ and in case of splitting functions, $N_{D U}^{B S}=1$. The power and cost values are provided in Tables III and IV and other simulation parameters for the network and GA algorithm are summarized in Table V.

Table V: Simulation Parameters

\begin{tabular}{llll}
\hline Simulation Parameters & Value & GA Parameters & Value \\
\hline Network area & $4 \mathrm{~km}^{2}$ & Mutation probability $p_{m}$ & 0.05 \\
Inter-site distance & $500 \mathrm{~m}$ & Crossover probability $p_{c}$ & 0.8 \\
Green field factor $\alpha$ & {$[0,1]$} & Number of individuals $N_{p}$ & 500 \\
Discount rate $b$ & $10 \%$ & Number of iterations & 100 \\
$D_{\text {cpri }}$ & $20 \mathrm{~km}$ & Absolute $\eta$ & $5 \%$ \\
$D_{\text {split }}$ & $2 \mathrm{~km}$ & $B$ & 100 \\
\hline \hline
\end{tabular}

In Figure 5, the optimality gap between GA solution and optimal solution obtained from the IBM ILOG CPLEX is shown. Moreover, we compare the conventional C-RAN solutions with C-RAN with functional splitting for different network sizes, i.e., with 4,9 , and 25 cells. We assume that we can use a part of the optical infrastructure which is already deployed, and we model this by multiplying the fiber costs by a factor $\alpha$, which is between 0 and 1 . The case $\alpha=1$ corresponds to greenfield fiber deployment. For small network size, the GA solution coincides with the optimal solution, however, as the network dimension scales, the optimality gap increases. This happens because with the increase of the network size, the

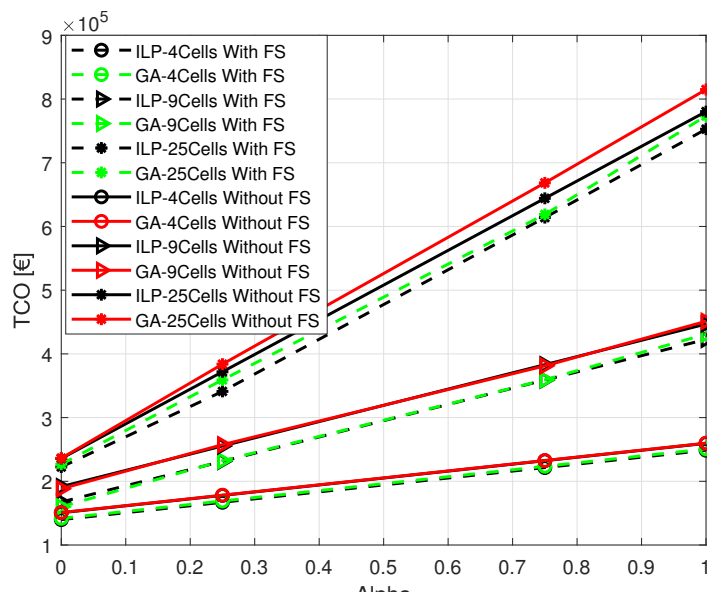

Figure 5: Comparison of GA solutions with ILP optimal solution [25]. $\alpha$ represents the greenfield deployment share, $\alpha=1$ means greenfield, $\alpha=0$ means brownfield, and FS means functional splitting.

size of search space increases and hence it becomes harder for the algorithm to find the optimal solution. When functional splitting is implemented, the DUs at the RU side should be activated and hence it adds extra expenses on the network. On the other hand, it can decrease the cost due to 1) less number of required equipment, 2) less fiber leasing cost due to less required capacity in the architecture, and 3) less power consumption at the cloud in the network. The major factor that differentiates between the cost of conventional C-RAN and C-RAN with functional splitting is the amount of utilized fiber. For the small network size, less amount of fiber is utilized. Therefore, the TCO of these two scenarios are very close. However, for larger network size, the TCO for CRAN with functional split is lower because the saving due to less utilization of fiber and equipment is greater than the cost increase due to extra power consumption.

\section{A. Migration Cost of C-RAN}

For the remaining results GA is used. We consider a $4 \mathrm{~km}^{2}$ network of 25 cells with $0.5 \mathrm{~km}$ inter-site distance. Figure 6 illustrates the TCO of conventional C-RAN, C-RAN with splitting, and D-RAN over time, with TCO minimization, for greenfield pool deployment. The TCO is shown as a function of the number of years after the architecture deployment. When the greenfield fiber deployment is assumed, it takes about 10 years for the TCO of C-RAN to become lower than that of D-RAN. This period of time is defined as crossover time in the remainder of this paper. Due to the sub-optimality of GA solutions, crossover times, i.e., 10 years in this case, are actually illustrating the worst case scenario. The optimum times can be earlier than these. Furthermore, the crossover time is decreased when brownfield fiber deployment is assumed. Specifically, it drops down to approximately four years when $\alpha=0.25$. Therefore, the optical infrastructure plays a determinant role in the migration process from D-RAN to C-RAN. The reader can refer to [25] for a deeper analysis under different deployment scenarios and cell sizes.

To show the impact of functional splitting, TCO for C-RAN with functional splitting is plotted in Figure 6. Since the RU 


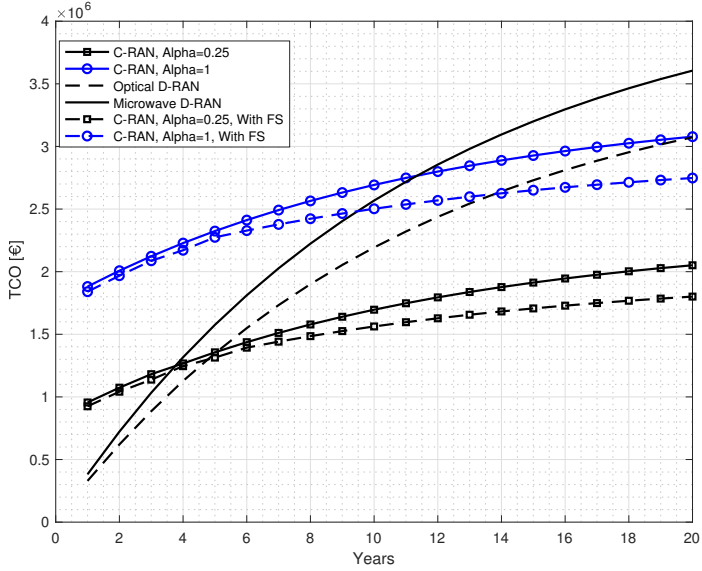

Figure 6: TCO of C-RAN and D-RAN over the years, with TCO minimization, for greenfield pool deployment and different $\alpha$, with and without FS.

sides have processing units, the network faces with higher costs incurred by extra power consumption and benefits from less required capacity and equipment in the architecture. TCO of C-RAN with functional splitting is lower that conventional C-RAN, because C-RAN with functional splitting has much lower OpEx while the CapEx are similar. Lower OpEx in C-RAN with functional splitting means lower annual costs and hence a more gentle slope in TCO increase compared to the conventional C-RAN. For fiber-rich operators, who already have fiber architecture in their backhaul network, i.e., brownfield fiber deployment, migrating to the conventional C-RAN is a logical choice with a reasonable crossover time of about 4 years. Such operator will not gain much from enabling functional splitting in a short term perspective. On the other hand, for fiber-short operators, which have to invest on deploying fiber in their network, i.e., greenfield fiber deployment, migrating to the conventional C-RAN with functional splitting is a better option, since the crossover time is about 2 years less than the conventional C-RAN.

Figure 7a depicts the breakdown of TCO and electric bills for green/brown field C-RAN with/without functional splitting and optical/microwave D-RAN. We keep the assumption of $\alpha=0.25$ for brownfield fiber deployment. The initial investment of greenfield fiber deployment is much higher than that of brownfield deployment but it gives us more flexibility to design the optimal fronthaul with lower OpEx. With enabling functional splitting, TCO is decreased since the OpEx and fiber leasing cost is reduced. Compared to D-RAN, both greenfield and brownfield fiber deployment require more initial investment. However, this cost is paid to decrease the OpEx. The reduction in OpEx means reduction in annual cost and hence after a certain crossover time (in case of brownfield fiber deployment about 4 years), C-RAN becomes an economically viable solution.Figure $7 \mathrm{~b}$ illustrates the electricity cost of $\mathrm{C}$ RAN and microwave/fiber D-RAN. For C-RAN, the cost of DUs is dropped dramatically due to the multiplexing gain introduced by C-RAN. In fact, the energy consumption of a base station is composed of energy consumption of DUs and RUs. In C-RAN the energy consumption of all DUs is partly included in the DU pool energy consumption and due to the

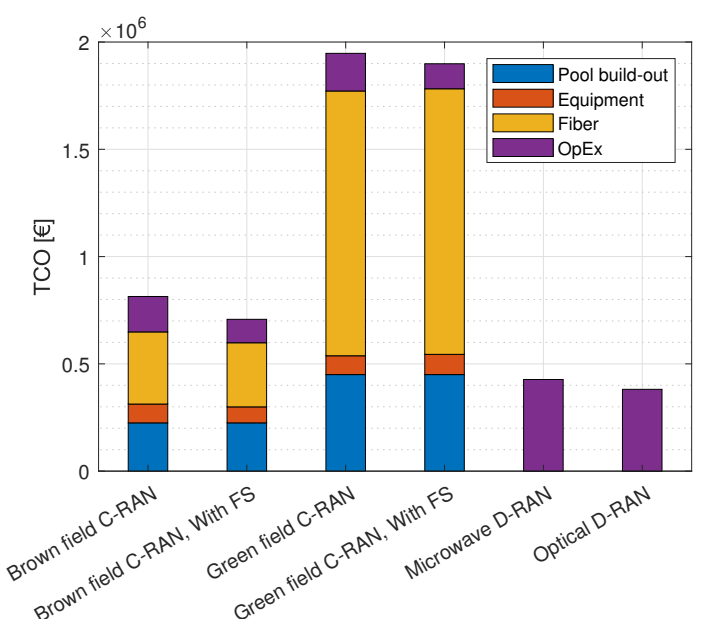

(a) TCO breakdown

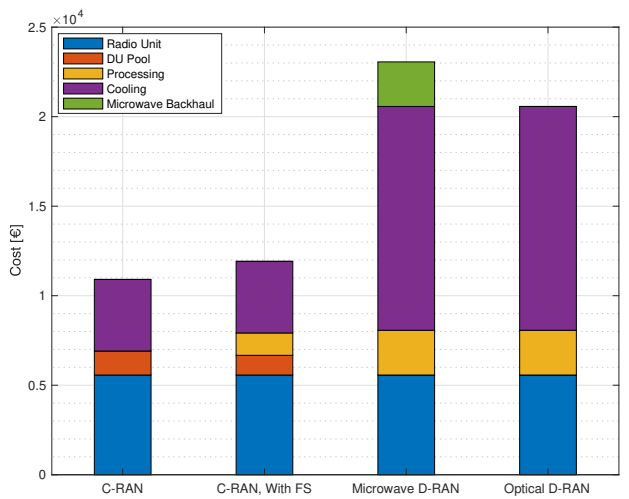

(b) Electricity cost breakdown

Figure 7: TCO and electric bills of C-RAN, Microwave/Optical D-RAN.

multiplexing gain, less DUs are active and more power is saved. In the network with the functional splitting, the RU side has the additional power consumption of activated DUs and hence consumes more power compared to the conventional C-RAN. For energy consumption of microwave D-RAN, there is the additional cost of microwave backhauling which makes this architecture less power and cost efficient over time. Therefore, in terms of electric bills, C-RAN is much more efficient than D-RAN. However, the initial TCO of C-RAN is higher due to having higher CapEx.

\section{B. Upgradability of $C-R A N$}

To investigate the upgradability of C-RAN, a simplified scenario is considered where 5 new cells need to be deployed after a period of four years. The locations of the new cells are given as input of the optimization problem. However, the simulations are run for different input locations and the results averaged in order to consider all possible locations in the area. In D-RAN, the upgrade process implies adding new cells and connections to the backhaul. We define two different upgrade scenarios, namely, preventive upgrade and later upgrade.

In preventive upgrade, the initial C-RAN deployment takes into account the extra cells that will be required in future, e.g., at year four. In other words, a margin is introduced in the 


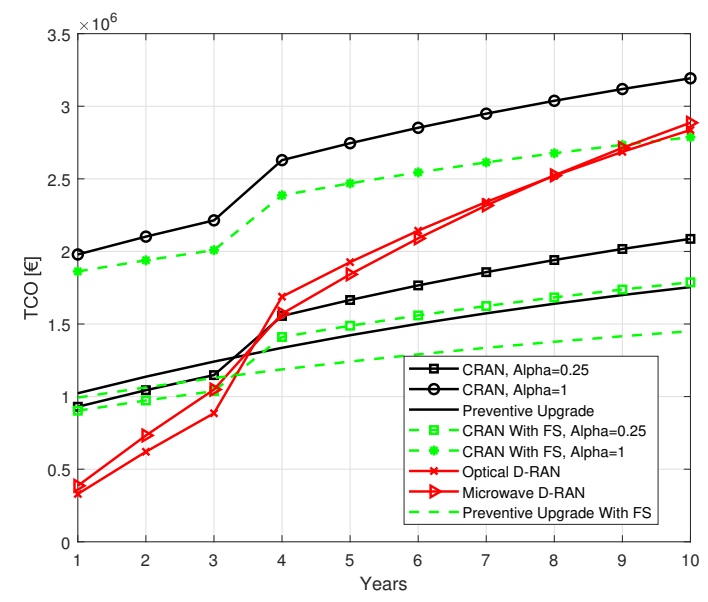

Figure 8: TCO of C-RAN with greenfield pool deployment, for no upgrade, later upgrade, and preventive upgrade with $\alpha=0.25$.

initial dimensioning of the system, i.e. pools and splitters are deployed with a buffer capacity to be utilized during future upgrades. In the later upgrade, we upgrade the network when it is required, e.g., after four years. The later upgrade is compared to the preventive upgrade, and D-RAN upgrade to identify the best strategy in terms of TCO.

Figure 8 compares the greenfield and brownfield fiber deployment with later and preventive upgrade. It shows that preventive upgrade is more cost-efficient than a later upgrade. In the beginning, the TCO of preventive upgrade is higher, since the network is underutilized. In fact, the preventive upgrade DU pool optimization problem and the fronthaul design is sub-optimal, however, after the upgrade time, the fronthaul design becomes optimal. The crossover time is in between year three and four, which is the year of upgrading. Brownfield pool deployment has less build-out costs but provides pools with lower capacity, and hence more pools are required to be deployed. As a result, equipment, maintenance, and electric costs increase. Although greenfield pool deployment requires build-out costs, pools have higher capacity, and lower number of pools and maintenance costs are required. Therefore, in the long term greenfield pool deployment becomes a better solution than brownfield pool deployment, in terms of TCO. Moreover, enabling functional splitting has lower TCO compared to the conventional C-RAN and it decreases the crossover time by one year in case brownfield fiber deployment.

\section{Sensitivity Analysis of Architectures}

In this section we analyze the impact of varying the electricity price, renting and leasing cost on the TCO. Figure 9 depicts the TCO fluctuation as the mentioned parameters are changed. The parameters that have the greatest impact on the value of the TCO are investigated in [20]. We doubled and halved the value of the electricity price and renting and leasing, then we calculated the new TCO. The blue dots represent the baseline results, e.g., the expected costs without any price/cost change, for each scenario. The vertical black lines show the variation of TCO when the values are changed. In the case of optical C-RAN, the infrastructure cost related to digging and rolling out fibers or leasing has the highest share in the TCO while in D-RAN, power consumption of running the network and equipment costs are the most expensive elements.

In case of electricity price, greenfield C-RAN has the least and microwave D-RAN has the most variation. The former is due to the fact that the architecture has been designed efficiently and hence it has the minimum OpEx and network component. The latter is because it includes all network components of D-RAN plus the microwave backhauling equipment. In the case of brownfield C-RAN, since the pools have less capacity, they require more equipment and it comes at the cost of more energy consumption and hence more sensitivity on energy cost. Therefore, microwave D-RAN is the most sensitive and greenfield C-RAN is the least sensitive architecture among all the scenarios to the variation of investigated parameters, i.e., energy price and renting and leasing cost. Compared to conventional C-RAN, C-RAN with splitting is more sensitive to the change of electricity price, because it consumes more energy due to the existence of functional processing at the RU side. Nevertheless, it is still less sensitive to the change of electricity price compared to non centralized deployment scenarios.

Another parameter to investigate is the leasing and renting costs. In fact, the price of leasing fibers, which varies depending on the region, has significant impact on the TCO. On the right hand side of Figure 9, it can be seen that D-RAN based architectures are quite sensitive to the renting costs, because the number of pools for the D-RAN architecture is high and hence the renting price has a considerable share of TCO. In case of C-RAN architectures, since the leasing and renting have very small share of TCO, the TCO is not as sensitive to change of this value as in D-RAN. From Figure 9, it can be inferred that sensitivity analysis is crucial in TCO analysis since the cost parameters' fluctuations can vary the TCO dramatically and hence can change the solution profitability. Therefore, sensitivity analysis should be taken into account together with TCO analysis in architecture design and cost analysis.

\section{CONCLUSIONS}

In this paper, we investigated a cost-efficient migration from D-RAN to C-RAN with TWDM-PON fronthaul with the functional splitting feature. We proposed an ILP optimization problem to minimize the TCO of C-RAN network. We took into account the practical limitations of the optical network such as functional splitting, fronthaul capacity requirements, CPRI limitations, capacity of splitters, wavelengths, and digital units. In the optimization problem, we found the optimal location of DU pools to minimize the TCO. Moreover, we investigated the upgradability of the network when it requires extension for more coverage and capacity. We initially solved the problem optimally using IBM ILOG CPLEX. Since the solutions of the ILP problems are not scalable, we extend the study and solve the problem with a heuristic algorithm, i.e., genetic algorithm. We showed that, for small network size, the heuristic algorithm could obtain the optimal solution; however, for larger network size, the optimality gap became non-zero. Then, we assessed the migration cost of C-RAN compared to two D-RAN 

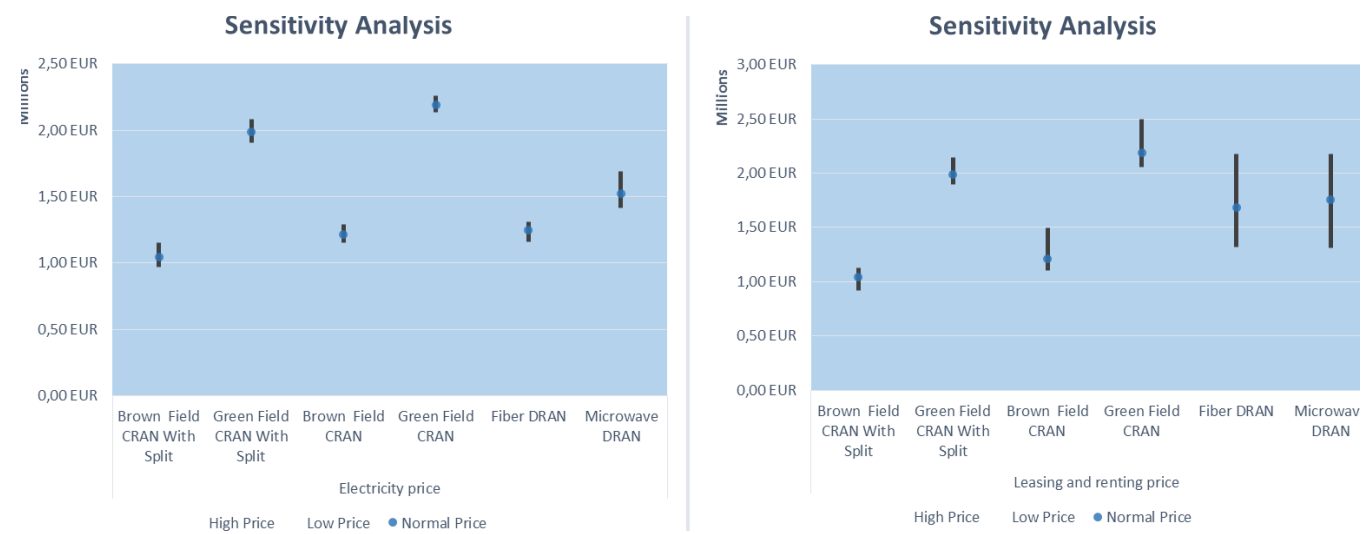

Figure 9: Sensitivity analysis of TCO with regards to the electricity and leasing/renting price change

architectures with microwave and optical backhaul. We showed that the fiber-rich operators should migrate to conventional CRAN while for fiber-short operators migrating to the C-RAN with functional splitting is a more reasonable choice. Our study showed that C-RAN with functional splitting had lower TCO and crossover time compared to C-RAN. Furthermore, in terms of upgradability, C-RAN was more cost efficient compared to D-RAN. Moreover, a preventive upgrade was more cost efficient compared to a later upgrade. Finally, we studied the sensitivity TCO with regards to the electricity and leasing/renting price change, and we showed that C-RAN architecture was less sensitive to the price change.

\section{REFERENCES}

[1] China Mobile Research Institute, "C-RAN the road towards green RAN," White Paper, October 2011.

[2] —, "Next generation fronthaul interface," White Paper, June 2015.

[3] Wang et al., "Cloud RAN architectures with optical and mm-wave transport technologies," in 19th International Conference on Transparent Optical Networks, ICTON 2017. IEEE, 2017.

[4] Chih-Lin et al., "Recent progress on C-RAN centralization and cloudification," IEEE Access, vol. 2, pp. 1030-1039, 2014.

[5] Pliatsios et al., "Realizing 5G vision through cloud RAN: technologies, challenges, and trends," EURASIP Journal on Wireless Communications and Networking, vol. 2018, no. 1, p. 136, May 2018. [Online]. Available: https://doi.org/10.1186/s13638-018-1142-1

[6] Masoudi et al., "Green mobile networks for 5G and beyond," IEEE Access, vol. 7, pp. $107270-107299,2019$.

[7] "Functional splits and use cases for small cell virtualization," Small Cell Forum release, Tech. Rep., 2016.

[8] Sriram et al., "Joint functional splitting and content placement for green hybrid CRAN," in 2019 IEEE International Symposium on Personal, Indoor and Mobile Radio Communications, Istanbul, Turkey, 2019.

[9] Matoussi et al., "A user centric virtual network function orchestration for agile 5G Cloud-RAN," in 2018 IEEE International Conference on Communications (ICC). IEEE, 2018

[10] Carapellese et al., "Energy-efficient baseband unit placement in a fixed/mobile converged WDM aggregation network," IEEE J. Select. Areas Commun., vol. 32, no. 8, pp. 1542-1551, Aug. 2014.

[11] Asensio et al., "Study of the centralization level of optical networksupported cloud RAN," in 2016 International Conference on Optical Network Design and Modeling (ONDM), May 2016.

[12] Tanaka and Agata, "Next-generation optical access networks for C-RAN," in Optical Fiber Communication Conference, Mar. 2015.

[13] Peng et al., "Fronthaul-constrained cloud radio access networks: insights and challenges," IEEE Wireless Commun., vol. 22, no. 2, pp. 152-160, Apr. 2015.

[14] Chen et al., "Backhauling in heterogeneous cellular networks: Modeling and tradeoffs," IEEE Transactions on Wireless Communications, vol. 14, no. 6, pp. 3194-3206, 2015.
[15] Widaa Ahmed et al., "Techno-economics of green mobile networks considering backhauling," in European Wireless 2014; 20th European Wireless Conference, May 2014.

[16] Mahloo et al., "Cost modeling of backhaul for mobile networks," in Communications Workshops (ICC), 2014 IEEE International Conference on. IEEE, 2014, pp. 397-402.

[17] Machuca et al., "Methodology for a cost evaluation of migration toward NGOA networks," IEEE/OSA Journal of Optical Communications and Networking, vol. 5, no. 12, pp. 1456-1466, 2013.

[18] Peralta et al., "Optimal scalability of fiwi networks based on multistage stochastic programming and policies," Journal of Optical Communications and Networking, vol. 9, no. 12, pp. 1172-1183, 2017.

[19] Wang et al., "Techno-economic analysis of active optical network migration toward next-generation optical access," IEEE/OSA Journal of Optical Communications and Networking, vol. 9, no. 4, pp. 327-341, 2017.

[20] Yaghoubi et al. "A techno-economic framework for 5G transport networks," IEEE wireless communications, vol. 25, no. 5, pp. 56-63, 2018.

[21] Frank et al., "Resource analysis and cost modeling for end-to-end 5g mobile networks," in Optical Network Design and Modelling (ONDM), 102019.

[22] Harutyunyan and Riggio, "How to migrate from operational lte/lte-a networks to c-ran with minimal investment?" IEEE Transactions on Network and Service Management, vol. 15, no. 4, pp. 1503-1515, Dec 2018.

[23] Wang et al., "Centralize or distribute? a techno-economic study to design a low-cost cloud radio access network," in 2017 IEEE International Conference on Communications (ICC). IEEE, 2017.

[24] Ranaweera et al., "Optical transport network design for 5G fixed wireless access," Journal of Lightwave Technology, vol. 37, no. 16, pp. 3893-3901, Aug 2019.

[25] Lisi et al., "Cost-effective migration towards C-RAN with optimal fronthaul design," 2017 IEEE International Conference on Communications (ICC), May 2017.

[26] Ericsson $\mathrm{AB}$ et al., "Common public radio interface (CPRI); interface specification v7.0," Huawei Technologies Co. Ltd, NEC Corporation, Alcatel Lucent, and Nokia Networks, 2015.

[27] Andrade et al., "Optimization of long-reach TDM/WDM passive optical networks," Optical Switching and Networking, vol. 16, pp. 36-45, Apr. 2015.

[28] Auer et al., "How much energy is needed to run a wireless network?" IEEE Wireless Commun., vol. 18, no. 5, pp. 40-49, Oct. 2011.

[29] Verbrugge et al., "Practical steps in techno-economic evaluation of network deployment planning part 1: methodology overview," in Telecommunications Network Strategy and Planning Symposium. The 13th International. IEEE, 2008, pp. 1-101.

[30] Schenk, "Valuation of flexibility for public investments," in Infrastructure Systems and Services: Building Networks for a Brighter Future (INFRA), 2008 First International Conference on. IEEE, 2008.

[31] Andrade et al., "Cost models for baseband unit BBU hotelling: From local to cloud," in 2015 IEEE $4^{\text {th }}$ International Conference on Cloud Networking (CloudNet), Oct. 2015.

[32] Masoudi et al., "Energy and spectrum efficient resource allocation in two-tier networks: A multiobjective approach," in 2017 Wireles Communications and Networking Conference (WCNC). IEEE, 2017. 